\title{
An audit of unnecessary preoperative urea and electrolyte panel tests in patients undergoing major orthopaedic surgery at a quaternary South African hospital
}

\author{
Y Moodley, (i) SS Mashele (iD \\ Faculty of Health and Environmental Sciences, Central University of Technology, South Africa
}

Corresponding author, email: yoshan@hotmail.com

\begin{abstract}
Background: Preoperative urea and electrolyte (U\&E) panels are frequently requested for major surgery patients at risk for postoperative acute kidney injury (AKI). There is only one published study that has audited unnecessary preoperative U\&E test panel utilisation in major surgery patients at a South African (SA) public-sector hospital. This has significant implications for laboratory workloads, healthcare expenditure, and patient-friendly practice in the resource-limited SA public healthcare sector.
\end{abstract}

Objective: To audit preoperative U\&E panel requests in a sample of SA patients undergoing major orthopaedic surgery.

Methods: We conducted a retrospective audit of adult primary hip arthroplasty patients who attended a quaternary SA hospital. Data on demographics, medical history, preoperative anaesthetic evaluations, operation details, and U\&E panel requests were collected from each patient's medical chart. The National Institute for Health and Care Excellence (NICE) guidelines, based on American Society of Anesthesiologists (ASA) grading and the presence of AKI risk factors, was used to distinguish between necessary and unnecessary preoperative $U \& E$ requests. We used descriptive statistics to analyse our study data.

Results: Of the 175 patients comprising our study sample, 23 (13.1\%) had preoperative U\&E panels requested unnecessarily. All 23 patients were otherwise healthy and did not have any AKI risk factors.

Conclusion: A small proportion of preoperative U\&E test panels in our study sample of major orthopaedic surgery patients were deemed unnecessary. With that being said, there is still room for improvement in practices around preoperative U\&E panel requests, which could be achieved through educational, computerised, and audit feedback interventions.

Keywords: kidney function testing, preoperative period, unnecessary procedures

\section{Introduction}

Acute kidney injury (AKI) occurs postoperatively in 1-39\% of patients undergoing major surgery, ${ }^{1-3}$ and is associated with a higher risk of postoperative mortality and increased healthcare expenditure in this surgical population. ${ }^{2}$ A preoperative history of kidney impairment is amongst the most notable risk factors for postoperative AKI. ${ }^{3}$ As such, assessing kidney function has become an integral part of the preoperative work-up in major surgery patients who are at risk for AKI. ${ }^{4}$ The urea and electrolytes (U\&E) test panel, which analyses serum levels of urea, creatinine, sodium, and potassium, has become the most frequently used method for assessing kidney function. ${ }^{5}$ However, unnecessary preoperative U\&E testing in patients who are at very low or no risk for AKI can contribute to significant unwarranted expenditure, ${ }^{6}$ depending on the volume of patients for which the U\&E panel is unnecessarily requested. Unnecessary laboratory tests also place additional workloads on laboratory technologists, ${ }^{7}$ and can lead to less patient-friendly practice..$^{8,9}$ Audits of preoperative laboratory testing at SA public-sector hospitals are scarce, and there is only one published SA study that has audited unnecessary preoperative laboratory test utilisation. ${ }^{6}$ Given the potential implications for laboratory workloads, healthcare expenditure, and patient-friendly practice in the resourcelimited environment of the SA public healthcare sector, the objective of our study was to add to the sparse existing literature on this topic by conducting an audit of preoperative U\&E panel requests in a sample of patients undergoing major orthopaedic surgery at a quaternary SA public-sector hospital.

\section{Methods}

\section{Study design and setting}

Our study was a retrospective audit of preoperative U\&E testing involving a sample of major surgery patients attending the Inkosi Albert Luthuli Central Hospital (IALCH) in Durban, SA. IALCH is a quaternary-level, strictly referral-based healthcare facility which provides specialist medical and surgical services to public-sector patients in the KwaZulu-Natal province.

\section{Differentiation between necessary [routine] and unnecessary preoperative U\&E tests}

There were no formal preoperative testing guidelines being implemented at IALCH at the time that this manuscript was 
being written. International guidance provided by the National Institute for Health and Care Excellence (NICE) recommends that preoperative kidney function tests (i.e. U\&E panels) should be routinely requested for patients who are at risk for postoperative AKI. ${ }^{4}$ According to these guidelines, preoperative kidney function tests are considered necessary or routine for major surgery patients classified as American Society of Anesthesiologists Physical Status Classification System (ASA) grade IV - patients with severe systemic disease that is a constant threat to life; patients classified as ASA grade III - patients with severe systemic disease; patients classified as ASA grade II - patients with mild systemic disease; or patients classified as ASA grade I (generally healthy patients) who have AKI risk factors such as intraperitoneal surgery, existing kidney impairment, diabetes, heart disease, elderly age, liver disease, and nonsteroidal anti-inflammatory drug (NSAID) use. ${ }^{4,10}$ Preoperative kidney function tests are not recommended for patients classified as ASA grade I who do not have any AKI risk factors. ${ }^{4}$ We used the criteria outlined in the NICE preoperative testing guidelines to distinguish between necessary and unnecessary U\&E panel requests in our study.

\section{Data collection}

We collected the data for our study through a retrospective chart review. All U\&E testing was carried out by a SANASaccredited laboratory attached to IALCH. Once we had identified an eligible patient, we captured de-identified information on their demographics, medical history, preoperative anaesthetic evaluations (including ASA grading), operation details, and U\&E panel requests on a password-protected electronic spreadsheet. The specific source documents from where this information was obtained included patient admission/clerking notes, preoperative anaesthetic clinic notes, operative notes, and laboratory test reports.

\section{Eligibility criteria}

Our study sample consisted of adult (aged $\geq 18$ years old) patients who underwent primary hip arthroplasty at IALCH between 23 September 2014 and 28 July 2016. Joint replacement surgeries, such as primary hip arthroplasty, are considered to be a major surgical procedure. ${ }^{4}$ We identified these patients by screening the operating room lists during the study period. If patients had two hip operations during this time period, only the first operation was considered for our study. We excluded patients with missing ASA gradings, as this is a key variable used in the NICE preoperative testing guidelines to distinguish between necessary and unnecessary preoperative kidney function testing. ${ }^{4}$

\section{Data analysis}

We used R version 3.6.2 (R Foundation, Vienna) to perform our data analysis. Descriptive statistical methods were used to analyse the study data. We summarised the overall characteristics of the study sample as frequencies and percentages. We also determined the frequency and percentage of necessary and unnecessary U\&E panels in each ASA group.

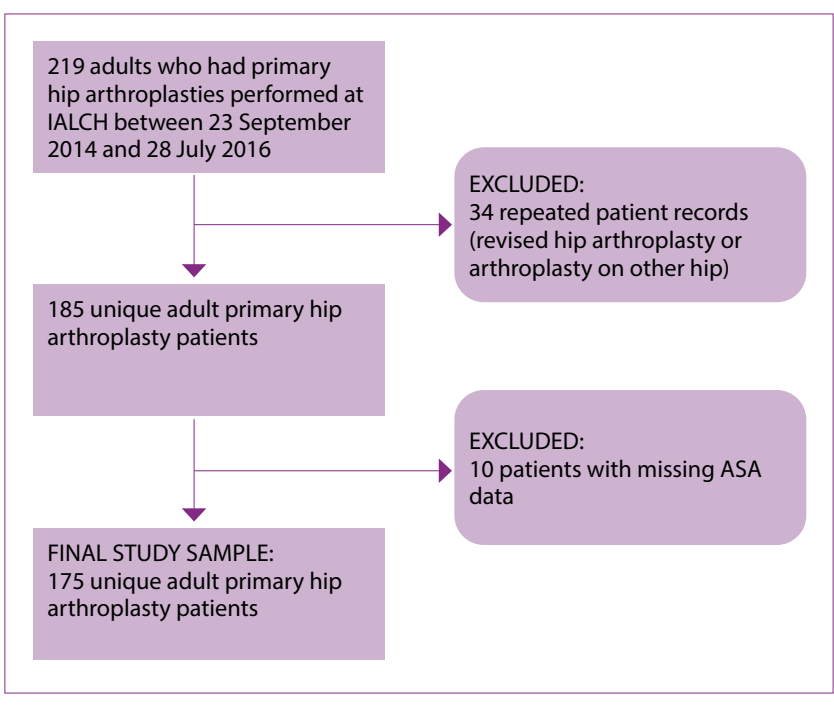

Figure 1: Derivation of the study sample

\section{Results}

An outline of the steps we took to derive our final study sample is provided in Figure 1. Following exclusion of repeat patient records and patients with missing data, our final study sample was comprised of 175 patients (Figure 1).

A summary of the demographic and medical characteristics in our study sample is shown in Table I. Just over one-quarter of our study sample was aged $\geq 65$ years old (48 patients, $27.4 \%$ ). The majority of our study sample were females (96 patients, 54.9\%). The three most common medical comorbidities were obesity ( 83 patients, 47.4\%), hypertension (81 patients, 46.3\%), and smoking (40 patients, $22.9 \%$ ). The three most common AKI risk factors were NSAID use (57 patients, $32.6 \%$ ), age $\geq 65$ years old (48 patients, $27.4 \%$ ), and diabetes (15 patients, $8.6 \%$ ). With regard to ASA grading, 31 patients were ASA grade I (17.7\%), 82 were ASA grade II (46.9\%), and 62 patients were ASA grade III/IV (35.4\%).

Patterns of U\&E panel requests in the study sample, stratified by ASA grade, are shown in Table II. Preoperative U\&E panels were ordered for all 175 patients in the study sample. In keeping with the NICE preoperative testing guidelines, U\&E panels were correctly requested as routine practice for $100 \%$ of patients classified as ASA grades II, III, or IV. Of the 175 patients comprising our study sample, there were 23 (13.1\%) who had U\&E panels unnecessarily requested. All 23 patients with unnecessary U\&E panels were in the ASA grade I group and accounted for $74.2 \%$ of patients in this group.

With regard to age distribution, U\&E panels were correctly requested for all patients aged $\geq 65$ years old (48 patients in the study sample) which was in full compliance with the NICE guidelines. Of the 127 patients aged < 65 years old, 56 patients (44.1\%) had at least one AKI risk factor which necessitated preoperative U\&E testing according to the NICE guidelines, while the remaining 71 patients (55.9\%) had unnecessary preoperative U\&E testing done (i.e. they did not meet any of the risk factor criteria for preoperative kidney function testing outlined in the NICE guidelines). 
Table I: Demographic, medical, and surgical characteristics of the study sample $(n=175)$

\begin{tabular}{|c|c|}
\hline Characteristic & $n(\%$ of $\mathrm{N})$ \\
\hline \multicolumn{2}{|l|}{ Age* } \\
\hline$<65$ years old & $127(72.6)$ \\
\hline$\geq 65$ years old & $48(27.4)$ \\
\hline \multicolumn{2}{|l|}{ Gender } \\
\hline Female & $96(54.9)$ \\
\hline Male & $79(45.1)$ \\
\hline \multicolumn{2}{|l|}{ Obesity } \\
\hline No & $92(52.6)$ \\
\hline Yes & $83(47.4)$ \\
\hline \multicolumn{2}{|l|}{ Hypertension } \\
\hline No & $94(53.7)$ \\
\hline Yes & $81(46.3)$ \\
\hline \multicolumn{2}{|l|}{ Diabetes* } \\
\hline No & $160(91.4)$ \\
\hline Yes & $15(8.6)$ \\
\hline \multicolumn{2}{|l|}{ Heart disease* } \\
\hline No & $165(94.3)$ \\
\hline Yes & $10(5.7)$ \\
\hline \multicolumn{2}{|l|}{ HIV } \\
\hline No & $137(78.3)$ \\
\hline Yes & $38(21.7)$ \\
\hline \multicolumn{2}{|l|}{ Liver disease* } \\
\hline No & $175(100.0)$ \\
\hline Yes & $0(0.0)$ \\
\hline \multicolumn{2}{|l|}{ Smoker } \\
\hline No & $135(77.1)$ \\
\hline Yes & $40(22.9)$ \\
\hline \multicolumn{2}{|c|}{ Renal impairment* } \\
\hline No & $163(93.1)$ \\
\hline Yes & $12(6.9)$ \\
\hline \multicolumn{2}{|l|}{ NSAIDs* } \\
\hline No & $118(67.4)$ \\
\hline Yes & $57(32.6)$ \\
\hline \multicolumn{2}{|l|}{ ASA } \\
\hline Grade I & $31(17.7)$ \\
\hline Grade II & $82(46.9)$ \\
\hline Grade III or IV & $62(35.4)$ \\
\hline \multicolumn{2}{|c|}{ Emergency surgery* } \\
\hline No & $173(98.9)$ \\
\hline Yes & $2(1.1)$ \\
\hline \multicolumn{2}{|c|}{ Intraperitoneal surgery* } \\
\hline No & $175(100.0)$ \\
\hline Yes & $0(0.0)$ \\
\hline
\end{tabular}

*Risk factors for AKI. Renal impairment defined as a preoperative estimated glomerular filtration rate $<60 \mathrm{~mL} / \mathrm{min} / 1.73 \mathrm{~m}^{2}$

\section{Discussion}

Overall, only a small proportion of preoperative U\&E panels in our study (13.1\%) were unnecessarily requested. The proportion of unnecessary preoperative U\&E panels in our study is lower than that reported in a study by Buley et al. (66.4\%) which involved patients undergoing various major surgical procedures at a regional hospital in Pietermaritzburg, SA. ${ }^{6}$

There are three possible explanations for the difference in unnecessary preoperative U\&E panel requests between our study and that conducted by Buley and colleagues. Firstly, the hospital-specific guidelines used by Buley et al., ${ }^{6}$ to establish the appropriateness of preoperative U\&E panels, did not consider ASA status which includes highly prevalent comorbid diseases, such as hypertension, which are not described as AKI risk factors in the evidence-based NICE preoperative testing guidelines. Thus, an overestimation of postoperative AKI risk at the Pietermaritzburg hospital, based on the hospital-specific guidelines, might have triggered overutilisation of preoperative U\&E testing. Secondly, the study by Buley et al. ${ }^{6}$ was conducted at a lower-level facility when compared with our specialist facility. Lower-level facilities host the majority of trainee anaesthesiologists and trainee surgeons in the SA public healthcare sector. It is possible that these less experienced anaesthesiologists and surgeons might be more likely to request unnecessary preoperative tests. ${ }^{11}$ Lastly, the majority of patients who attend higher-level facilities, such as IALCH, are complex cases that require specialised care. ${ }^{12}$ These patients are likely to have existing mild-to-severe systemic disease (ASA grade II-IV), which would automatically qualify them for routine preoperative U\&E testing according to the NICE preoperative testing guidelines. Indeed, $82.3 \%$ of patients in our study sample were graded as ASA II-IV and all these patients correctly had preoperative U\&E test panels requested according to the NICE preoperative testing guidelines.

Although we report a small proportion of unnecessary preoperative U\&E panel requests in our study, we believe that there is still room for improvement. There are several factors which impact anaesthesiologists' and surgeons' decisions on preoperative testing. These include level of experience, practice tradition, belief that other anaesthesiologists or surgeons want the test to be done, medicolegal worries, concerns that not doing the tests will result in the surgery being delayed or cancelled, and lack of awareness of appropriate evidence and preoperative testing guidelines. ${ }^{11,13}$ In response to these factors, attempts have been made to develop and use educational interventions (such as guidelines, seminars, posters to improve knowledge and awareness around preoperative testing), computerised interventions (for example, gatekeeping on

Table II: U\&E panel requests in the study sample $(\mathrm{N}=175)$, stratified by ASA grade

\begin{tabular}{lccc}
\hline ASA grade & $\begin{array}{c}\text { Total } \\
\boldsymbol{n}(\% \text { of } \mathbf{N})\end{array}$ & $\begin{array}{c}\text { Routine } \\
\boldsymbol{n}(\% \text { of total) }\end{array}$ & $\begin{array}{c}\text { Unnecessary } \\
\boldsymbol{n}(\% \text { of total) }\end{array}$ \\
\hline I & $31(17.7)$ & $8(25.8)$ & $23(74.2)$ \\
II & $82(49.6)$ & $82(100.0)$ & $0(0.0)$ \\
III or IV & $62(35.4)$ & $62(100.0)$ & $0(0.0)$ \\
\hline
\end{tabular}

According to the NICE preoperative testing guidelines, routine U\&E panels are necessary/routine for patients classified as ASA grades II-IV or patients classified as ASA grade I who have risk factors for AKI 
electronic laboratory request systems for patients who do not meet the criteria for preoperative testing), and audit feedback to improve clinical decision making around preoperative testing in anaesthesiologists and surgeons. ${ }^{9}$ It must be noted that although these interventions demonstrate some success in the short term, the long-term effectiveness of these interventions is questionable and likely requires persistent reinforcement.9,14

The main strengths of our study were its fairly large sample size for a facility-level audit; the completeness of the patient data (only $5 \%$ of potentially eligible patients were excluded due to missing ASA data); and the long audit period, of almost two years, which would have accounted for changes in overall preoperative testing preferences amongst anaesthesiologists and surgeons at IALCH due to staff turnover.

The main limitation of this study was that the NICE preoperative testing guidelines are strongly based on ASA grading, which is subjective amongst anaesthesiologists. ${ }^{15}$ However, IALCH is a quaternary-level facility and most of the anaesthesiologists practising at the hospital are already highly experienced, senior specialists. Therefore, it is likely that the ASA assessments made by the senior anaesthesiologists at IALCH are far more reliable than those made at lower-level facilities which host more trainee anaesthesiologists. Another limitation is that our study findings might not be completely generalisable to major surgery populations in other SA hospital settings as our study was conducted at a single, quaternary-level facility and only involved patients undergoing major orthopaedic surgery. Lastly, we acknowledge that our data is four years old, and that it might not reflect current practices in the hospital. Due to restrictions on non-essential research in the hospital at the time of writing this article (as a result of the COVID-19 pandemic), we were unable to update our dataset beyond 2016. We recommend that future studies on this topic address this limitation and the other limitations we have listed.

\section{Conclusion}

A small proportion of preoperative U\&E test panels in our study sample of major orthopaedic surgery patients were unnecessarily requested. We acknowledge that despite the small proportion of unnecessary preoperative U\&E tests observed in our study sample, there is still room for improvement in practices around preoperative U\&E panel requests. We recommend that interventions that seek to improve preoperative U\&E panel utilisation be implemented and persistently reinforced amongst anaesthesiologists and surgeons at our hospital to further reduce unnecessary preoperative U\&E requests.

\section{Conflict of interest}

The authors declare no conflict of interest.

\section{Funding source}

None.

\section{Ethical approval}

Our study was part of a larger healthcare utilisation audit which was approved by the Biomedical Research Ethics Committee at the University of KwaZulu-Natal, SA (Protocol BE595/16).

\section{ORCID}

Y Moodley (iD https://orcid.org/0000-0002-4119-1734

SS Mashele (iD) https://orcid.org/0000-0002-6339-4807

\section{References}

1. Lei VJ, Luong T, Shan E, et al. Risk stratification for postoperative acute kidney injury in major noncardiac surgery using preoperative and intraoperative data. JAMA Network Open. 2019;2(12):e1916921. https://doi.org/10.1001/ jamanetworkopen.2019.16921.

2. Hobson C, Ozrazgat-Baslanti T, Kuxhausen A, et al. Cost and mortality associated with postoperative acute kidney injury. Ann Surg. 2015;261(6):1207-14. https:// doi.org/10.1097/sla.0000000000000732.

3. Kheterpal S, Tremper KK, Heung M, et al. Development and validation of an acute kidney injury risk index for patients undergoing general surgery: results from a national data set. Anesthesiology. 2009;110(3):505-15. https://doi. org/10.1097/ALN.0b013e3181979440.

4. Routine preoperative tests for elective surgery. National Institute for Health and Care Excellence; 2016. Available from: https://www.nice.org.uk/guidance/ng45/ chapter/Recommendations\#table-3-major-or-complex-surgery.

5. Blann A. Why do we test for urea electrolytes? Nurs Times. 2014;110(5):19-21.

6. Buley HE, Bishop D, Rodseth R. The appropriateness of preoperative blood testing: A retrospective evaluation and cost analysis. S Afr Med J. 2015;105(6):487-90. https://doi.org/10.7196/samj.9318.

7. Kwok J, Jones B. Unnecessary repeat requesting of tests: an audit in a government hospital immunology laboratory. J Clin Pathol. 2005;58(5):457-62. https://doi.org/10.1136/jcp.2004.021691.

8. Bindraban RS, Van Beneden MLH, Kramer MHH, et al. A multicenter beforeafter study on reducing unnecessary diagnostics by changing the attitude of caregivers: Protocol for the RODEO Project. JMIR Res Protoc. 2018;7(8):e10473. https://doi.org/10.2196/10473.

9. Bindraban RS, Ten Berg MJ, Naaktgeboren CA, et al. reducing test utilization in hospital settings: a narrative review. Ann Lab Med. 2018;38(5):402-12. https:// doi.org/10.3343/alm.2018.38.5.402.

10. Acute kidney injury: prevention, detection and management. NICE Guideline NG148. National Institute for Health and Care Excellence; 2019. Available from: https://www.nice.org.uk/guidance/ng148/chapter/ Recommendations\#identifying-the-causes-of-acute-kidney-injury.

11. Ranasinghe P, Perera YS, Senaratne JA, et al. Preoperative testing in elective surgery: Is it really cost effective? Anesth Essays Res. 2011;5(1):28-32. https://doi. org/10.4103/0259-1162.84177.

12. Haeusler IL, Sajan M, Parrish A. Mind the message: Referral letter quality at a South African medical outpatient department. S Afr Med J. 2020;110(5):396-9. https://doi.org/10.7196/SAMJ.2020.v110i5.14275.

13. Brown SR, Brown J. Why do physicians order unnecessary preoperative tests? A qualitative study. Fam Med. 2011;43(5):338-43.

14. Axt-Adam P, Van der Wouden JC, Van der Does E. Influencing behavior of physicians ordering laboratory tests: a literature study. Med Care. 1993;31(9):78494. https://doi.org/10.1097/00005650-199309000-00003.

15. Daabiss M. American Society of Anaesthesiologists physical status classification. Indian J Anaesth. 2011;55(2):111-5. https://doi.org/10.4103/0019-5049.79879. 